\title{
Laryngeal Basaloid Carcinoma
}

National Cancer Institute

\section{Source}

National Cancer Institute. Laryngeal Basaloid Carcinoma. NCI Thesaurus. Code C54334.

An aggressive variant of squamous cell carcinoma that arises from the pyriform sinus or supraglottic area. It is characterized by the presence of small malignant cells with hyperchromatic nuclei and scant amount of cytoplasm forming lobules with peripheral palisading. Comedonecrosis may be present. An in situ or invasive keratinizing squamous cell carcinoma component is always present. Signs and symptoms include hoarseness, dysphagia, pain, and neck mass. 